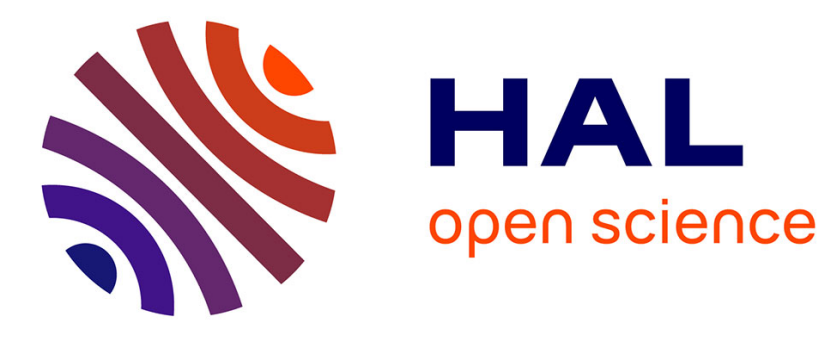

\title{
Symmetry Preserving Interpolation
}

Erick Rodriguez Bazan, Evelyne Hubert

\section{To cite this version:}

Erick Rodriguez Bazan, Evelyne Hubert. Symmetry Preserving Interpolation. ISSAC 2019 International Symposium on Symbolic and Algebraic Computation, Jul 2019, Beijing, China. 10.1145/3326229.3326247. hal-01994016

\section{HAL Id: hal-01994016 https://hal.science/hal-01994016}

Submitted on 25 Jan 2019

HAL is a multi-disciplinary open access archive for the deposit and dissemination of scientific research documents, whether they are published or not. The documents may come from teaching and research institutions in France or abroad, or from public or private research centers.
L'archive ouverte pluridisciplinaire HAL, est destinée au dépôt et à la diffusion de documents scientifiques de niveau recherche, publiés ou non, émanant des établissements d'enseignement et de recherche français ou étrangers, des laboratoires publics ou privés. 


\section{Symmetry Preserving Interpolation}

\author{
Erick Rodriguez Bazan \\ Université Côte d'Azur, France \\ Inria Méditerranée, France \\ erick-david.rodriguez-bazan@inria.fr
}

\author{
Evelyne Hubert \\ Université Côte d'Azur, France \\ Inria Méditerranée, France \\ evelyne.hubert@inria.fr
}

\begin{abstract}
The article addresses multivariate interpolation in the presence of symmetry. Interpolation is a prime tool in algebraic computation while symmetry is a qualitative feature that can be more relevant to a mathematical model than the numerical accuracy of the parameters. The article shows how to exactly preserve symmetry in multivariate interpolation while exploiting it to alleviate the computational cost. We revisit minimal degree and least interpolation with symmetry adapted bases, rather than monomial bases. This allows to construct bases of invariant interpolation spaces in blocks, capturing the inherent redundancy in the computations. We show that the so constructed symmetry adapted interpolation bases alleviate the computational cost of any interpolation problem and automatically preserve any equivariance of this interpolation problem might have.
\end{abstract}

\section{KEYWORDS}

Interpolation, Symmetry, Representation Theory, Group Action

\section{INTRODUCTION}

Preserving and exploiting symmetry in algebraic computations is a challenge that has been addressed within a few topics and, mostly, for specific groups of symmetry $[2,7,8,10,11,13-16,18$, $19,22]$. The present article addresses multivariate interpolation in the presence of symmetry. Due to its relevance in approximation theory and geometrical modeling, interpolation is a prime topic in algebraic computation. Among the several problems in multivariate interpolation $[9,17]$, we focus on the construction of a polynomial interpolation space for a given set of linear forms. Assuming the space generated by the linear forms is invariant under a group action, we show how to, not only, preserve exactly the symmetry, but also, exploit it so as to reduce the computational cost.

For a set of $r$ points $\xi_{1}, \ldots, \xi_{r}$ in $n$-space, and $r$ values $\eta_{1}, \ldots, \eta_{r}$ the basic interpolation problem consists in finding a $n$-variate polynomial function $p$ such that $p\left(\xi_{i}\right)=\eta_{i}$, for $1 \leq i \leq r$. The evaluations at the points $\xi_{i}$ form a basic example of linear forms. The space they generate is invariant under a group action when the set of points is a union of orbits of this group action. A first instance of symmetry is invariance. The above interpolation problem is invariant if $\eta_{i}=\eta_{j}$ whenever $\xi_{i}$ and $\xi_{j}$ belong to the same orbit. It is then natural to expect an invariant polynomial as interpolant. Yet, contrary to the univariate case, there is no unique interpolant of minimal degree and the symmetry of the interpolation problem may very well be violated (compare Figure 2 and 1).

In this article we shall consider a general set of linear forms, invariant under a group action, and seek to compute interpolants that respect the symmetry of the interpolation problem. We mentioned invariance as an instance of symmetry, but equivariance is the more general concept. An interpolation space for a set of linear forms is a subspace of the polynomial ring that has a unique interpolant for each instantiated interpolation problem. We show that the unique interpolants automatically inherit the symmetry of the problem when the interpolation space is invariant (Section 3).

A canonical interpolation space, the least interpolation space, was introduced in [3-5]. We shall review that it is invariant as soon as the space of linear forms is. In floating point arithmetics though, the computed interpolation space might fail to be exactly invariant. Yet, in mathematical modeling, symmetry is often more relevant than numerical accuracy. We shall remedy this flaw and further exploit symmetry to mitigate the cost and numerical sensitivity of computing a minimal degree or least interpolation space.

As other minimal degree interpolation spaces, the least interpolation space can be constructed by Gaussian elimination in a multivariate Vandermonde (or collocation) matrix. The columns of the Vandermonde matrix are indexed by monomials. We show how any other graded basis of the polynomial ring can be used. In particular there is a two fold gain in using a symmetry adapted basis. On one hand, the computed interpolation space will be exactly invariant independently of the accuracy of the data for the interpolation problem. On the other hand, the new Vandermonde matrix is block diagonal so that Gaussian elimination can be performed independently on smaller size matrices, with better conditioning. Further computational savings result from identical blocks being repeated according to the degree of the related irreducible representations of the group. Symmetry adapted bases also plaid a prominent role in $[2,11,19]$ where it allowed the block diagonalisation of a multivariate Hankel matrix.

In Section 2 we define minimal degree and least interpolation space and review how to compute a basis of it with Gaussian elimination. In Section 3 we make explicit how symmetry is expressed and the main ingredient to preserve it. In Section 4 we review symmetry adapted bases and show how the Vandermonde matrix becomes block diagonal in these. This is applied to provide an algorithm for the computation of invariant interpolation spaces in Section 5 together with a selection of relevant invariant and equivariant interpolation problems.

\section{POLYNOMIAL INTERPOLATION}

We review in this section the definitions and constructions of interpolation spaces of minimal degree. By introducing general dual polynomial bases we generalize the construction of least interpolation spaces. We shall then be in a position to work with adapted bases to preserve and exploit symmetry. 


\subsection{Interpolation space}

Hereafter, $\mathbb{K}$ denotes either $C$ or $\mathbb{R} . \mathbb{K}[\mathrm{x}]=\mathbb{K}\left[x_{1}, \ldots, x_{n}\right]$ denotes the ring of polynomials in the variables $x_{1}, \ldots, x_{n}$ with coefficients in $\mathbb{K} ; \mathbb{K}[\mathrm{x}]_{\leq \delta}$ and $\mathbb{K}[\mathrm{x}]_{\delta}$ the $\mathbb{K}$-vector spaces of polynomials of degree at most $\delta$ and the space of homogeneous polynomials of degree $\delta$ respectively.

The dual of $\mathbb{K}[\mathrm{x}]$, the set of $\mathbb{K}$-linear forms on $\mathbb{K}[\mathrm{x}]$, is denoted by $\mathbb{K}[\mathrm{x}]^{*}$. A typical example of a linear form on $\mathbb{K}[\mathrm{x}]$ is the evaluation $\mathbb{e} \xi$ at a point $\xi$ of $\mathbb{K}^{n}$. It is defined by

$$
\begin{array}{ccc}
\mathbb{e}_{\xi}: \mathbb{K}[\mathrm{x}] & \rightarrow & \mathbb{K} \\
p & \mapsto & p(\xi) .
\end{array}
$$

Other examples of linear forms on $\mathbb{K}[\mathrm{x}]$ are given by compositions of evaluation and differentiation

$$
\begin{aligned}
\Lambda: \mathbb{K}[\mathrm{x}] & \rightarrow \mathbb{K} \\
p & \mapsto \sum_{j=1}^{r} \mathbb{e}_{\xi_{j}} \circ q_{j}(\partial)(p),
\end{aligned}
$$

with $\xi_{j} \in \mathbb{K}^{n}, q_{j} \in \mathbb{K}[\mathrm{x}]$ and $\partial^{\alpha}=\frac{\partial}{\partial x_{1}^{\alpha_{1}}} \ldots \frac{\partial}{\partial x_{n}^{\alpha_{n}}}$.

Let $\xi_{1}, \ldots, \xi_{r}$ be a finite set of points in $\mathbb{K}^{n}$. Lagrange interpolation consists in finding, for any $\eta_{1}, \ldots \eta_{r} \in \mathbb{K}$, a polynomial $p$ such that $\mathbb{e}_{\xi_{j}}(p)=\eta_{j}, 1 \leq j \leq r$. More generally an interpolation problem is a pair $(\Lambda, \phi)$ where $\Lambda$ is a finite dimensional linear subspace of $\mathbb{K}[\mathrm{x}]^{*}$ and $\phi: \Lambda \longrightarrow \mathbb{K}$ is a $\mathbb{K}$-linear map. An interpolant, i.e., a solution to the interpolation problem, is a polynomial $p$ such that

$$
\lambda(p)=\phi(\lambda) \text { for any } \lambda \in \Lambda \text {. }
$$

An interpolation space for $\Lambda$ is a polynomial subspace $P$ of $\mathbb{K}[\mathrm{x}]$ such that Equation (1) has a unique solution in $P$ for any map $\phi$.

\subsection{Vandermonde matrix}

For $\mathcal{P}=\left\{p_{1}, p_{2}, \ldots, p_{m}\right\}$ and $\mathcal{L}=\left\{\lambda_{1}, \lambda_{2}, \ldots, \lambda_{r}\right\}$ linearly independent sets of $\mathbb{K}[\mathrm{x}]$ and $\mathbb{K}[\mathrm{x}]^{*}$ respectively, we introduce the (generalized) Vandermonde matrix

$$
\mathrm{W}_{\mathcal{L}}^{\mathcal{P}}:=\left[\lambda_{i}\left(p_{j}\right)\right]_{\substack{1 \leq i \leq r \\ 1 \leq j \leq m}}^{\substack{1 \leq j \\ \text {. }}}
$$

As in the univariate case, the Vandermonde matrix appears naturally in the interpolation problem. $\operatorname{span}_{\mathbb{K}}(\mathcal{P})$ is an interpolation space for $\operatorname{span}_{\mathbb{K}}(\mathcal{L})$ if and only if $\mathrm{W}_{\mathcal{L}}^{\mathcal{P}}$ is an invertible matrix. This leads to a straightforward approach to compute an interpolation space for $\langle\mathcal{L}\rangle$. Since the elements of $\mathcal{L}$ are linearly independents, there is $\delta>0$ such that $\mathrm{W}_{\mathcal{L}}^{\mathcal{P}_{\delta}}$ has full row rank, where $\mathcal{P}_{\delta}$ is a basis of $\mathbb{K}[\mathrm{x}]_{\leq \delta}$. For Lagrange interpolation $\delta \leq|\mathcal{L}|$. Hence we can choose $r$ linearly independent columns $j_{1}, j_{2}, \ldots j_{r}$ of $\mathrm{W}_{\mathcal{L}}^{\mathcal{P}_{\mathcal{S}}}$ and the corresponding space $P=\operatorname{span}_{\mathbb{K}}\left(p_{j_{1}}, \ldots p_{j_{k}}\right)$ is an interpolation space for $\Lambda$.

In order to select $r$ linearly independent columns of $\mathrm{W}_{\mathcal{L}}^{\mathcal{P}_{\delta}}$ we can use any rank revealing decomposition of $\mathrm{W}_{\mathcal{L}}^{\mathcal{P}_{\mathcal{S}}}$. Singular value decomposition (SVD) and $Q R$ decomposition provide better numerical accuracy but to obtain a minimal degree interpolation space we shall resort to Gauss elimination. It produces a LU factorization of $\mathrm{W}_{\mathcal{L}}^{\mathcal{P}_{\delta}}$ where $\mathrm{L}$ is an invertible matrix and $\mathrm{U}=\left[u_{i j}\right]_{\substack{1 \leq i \leq r \leq m \\ 1 \leq j \leq m}}$ is in row echelon form. This means that there exists an increasing sequence $j_{1}, \ldots, j_{r}$ with $j_{i} \geq i$, such that $u_{i j_{i}}$ is the first non-zero entry in the $i$-th row of $\mathrm{U}$. We call $j_{1}, \ldots, j_{r}$ the echelon index sequence of
$\mathrm{W}_{\mathcal{L}}^{\mathcal{P}_{\delta}}$. They index a maximal set of linearly independent columns of $\mathrm{W}_{\mathcal{L}}^{\mathcal{P}_{\delta}}$.

\subsection{Minimal degree}

It is desirable to build an interpolation space such that the degree of the interpolating polynomials be as small as possible. We shall use the definition of minimal degree solution for an interpolation problem defined in $[4,5,20]$.

Definition 2.1. An interpolation space $P$ for $\Lambda$ is of minimal degree if for any other interpolation space $Q$ for $\Lambda$

$$
\operatorname{dim}\left(Q \cap \mathbb{K}[\mathrm{x}]_{\leq \delta}\right) \leq \operatorname{dim}\left(P \cap \mathbb{K}[\mathrm{x}]_{\leq \delta}\right), \forall \delta \in \mathbb{N} .
$$

We say that a countable set of homogeneous polynomials $P=$ $\left\{p_{1}, p_{2}, \ldots\right\}$ is ordered by degree if $i \leq j$ implies that $\operatorname{deg} p_{i} \leq \operatorname{deg} p_{j}$.

Proposition 2.2. Let $\mathcal{L}$ be a basis of $\Lambda$. Let $\mathcal{P}_{\delta}, \delta>0$, be a homogeneous basis of $\mathbb{K}[\mathrm{x}]_{\leq \delta}$ ordered by degree, such that $\mathrm{W}_{\mathcal{L}}^{\mathcal{P}_{\delta}}$ has full row rank. Let $j_{1}, \ldots, j_{r}$ be the echelon sequence of $\mathrm{W}_{\mathcal{L}}^{\mathcal{P}_{\delta}}$ obtained by Gauss elimination with partial pivoting. Then $P:=\left\langle p_{j_{1}}, \ldots, p_{j_{r}}\right\rangle$ is a minimal degree interpolation space for $\Lambda$.

Proof. Let $Q$ be another interpolation space for $\Lambda$. Let $q_{1}, q_{2} \ldots q_{m}$ be a basis of $Q \cap \mathbb{K}[\mathrm{x}]_{\leq d}$ with $d \leq \delta$. Since $\mathcal{P}_{\delta}$ is a homogeneous basis of $\mathbb{K}[\mathrm{x}]_{\leq \delta}$, any $q_{i}$ can be written as a linear combination of elements of $\mathcal{P}_{\delta} \cap \mathbb{K}[\mathrm{x}]_{\leq d}$. Considering $q_{i}=\sum_{j} a_{j i} p_{j}$ we get that $\lambda\left(q_{i}\right)=\sum_{j} a_{j i} \lambda\left(p_{j}\right)$ for any $\lambda \in \Lambda$.

Let $\left\{p_{j_{i_{1}}}, p_{j_{i_{2}}}, \ldots p_{j_{i_{n}}}\right\}$ be the elements of $P$ that form a basis of $P \cap \mathbb{K}[\mathrm{x}]_{\leq d}$. Gauss elimination on $\mathrm{W}_{\mathcal{L}}^{\mathcal{P}_{\delta}}$ ensures that $\lambda(b)$ is a linear combination of $\lambda\left(p_{j_{i_{1}}}\right), \ldots \lambda\left(p_{j_{i_{n}}}\right)$ for any $b \in \mathcal{P}_{\delta} \cap \mathbb{K}[\mathrm{x}]_{\leq d}$ and $\lambda \in$ $\Lambda$. The latter implies that $\lambda\left(q_{i}\right)=\sum_{k=1}^{n} c_{k i} \lambda\left(p_{j_{i_{k}}}\right)$ for $1 \leq i \leq m$ and $c_{k i} \in \mathbb{K}$. If $m>n$ then the matrix $\mathrm{C}=\left(c_{i j}\right)_{\substack{1 \leq i \leq m \\ 1 \leq j \leq n}}$ has linearly independent columns, and therefore there exist $d_{1}, d_{2}, \ldots d_{m} \in \mathbb{K}$ such that $\sum_{i=1}^{m} d_{i} \lambda\left(q_{i}\right)=\lambda\left(\sum_{i=1}^{m} d_{i} q_{i}\right)=0$ for any $\lambda \in \Lambda$ which is a contradiction with the fact that $Q$ is an interpolation space of $\Lambda$. Then we can conclude that $m \leq n$ and $P$ is a minimal degree interpolation space for $\Lambda$.

\subsection{Duality and apolar product}

$\mathbb{K}[\mathrm{x}]^{*}$ can be identified with the ring of formal power series $\mathbb{K}[[\partial]]$ through the isomorphism $\Phi: \mathbb{K}[[\partial]] \longrightarrow \mathbb{K}[\mathrm{x}]^{*}$, where for $p=$ $\sum_{\alpha} p_{\alpha} x^{\alpha} \in \mathbb{K}[\mathrm{x}]$ and $f=\sum_{\alpha \in \mathbb{N}^{n}} f_{\alpha} \partial^{\alpha} \in \mathbb{K}[[\partial]]$

$$
\Phi(f)(p):=\sum_{\alpha \in \mathbb{N}^{n}} f_{\alpha} \frac{\partial^{\alpha} p}{\partial x^{\alpha}}(0)=\sum_{\alpha \in \mathbb{N}} \alpha ! f_{\alpha} p_{\alpha} .
$$

For instance, the evaluation $\mathbb{e}_{\xi}$ at a point $\xi \in \mathbb{K}^{n}$ is represented by $\mathrm{e}^{(\xi, \partial)}=\sum_{k \in \mathbb{N}} \frac{(\xi, \partial)^{k}}{k !}$, the power series expansion of the exponential function with frequency $\xi$. The dual pairing

$$
\begin{array}{cl}
\mathbb{K}[\mathrm{x}]^{*} \times \mathbb{K}[\mathrm{x}] & \rightarrow \mathbb{K} \\
(\lambda, p) & \rightarrow \lambda(p)
\end{array}
$$

brings the apolar product on $\mathbb{K}[\mathrm{x}]$ by associating $p \in \mathbb{K}[\mathrm{x}]$ to $p(\partial) \in$ $\mathbb{K}[[\partial]]$. For $p=\sum_{\alpha} p_{\alpha} x^{\alpha}$ and $q=\sum_{\alpha} q_{\alpha} x^{\alpha}$ the apolar product between $p$ and $q$ is given by $\langle p, q\rangle:=p(\partial) \bar{q}=\sum_{\alpha} \alpha ! p_{\alpha} \bar{q}_{\alpha} \in \mathbb{K}$. Note that for a linear map $a: \mathbb{K}^{n} \rightarrow \mathbb{K}^{n},\langle p, q \circ a\rangle=\left\langle p \circ \bar{a}^{t}, q\right\rangle$. 
For a set of linearly independent homogeneous polynomials $\mathcal{P}$ we define the dual set $\mathcal{P}^{\dagger}$ to be a set of homogeneous polynomials such that $\left\langle p_{i}^{\dagger}, p_{j}\right\rangle=\delta_{i j}$. For instance the dual basis of the monomial basis $\left\{x^{\alpha}\right\}_{\alpha \in \mathbb{N}^{n}}$ is $\left\{\frac{1}{\alpha !} x^{\alpha}\right\}_{\alpha \in \mathbb{N}^{n}}$. Thus any linear form $\lambda \in \mathbb{K}[\mathrm{x}]^{*}$ can be written as $\lambda=\sum_{\alpha \in \mathbb{N}^{n}} \frac{1}{\alpha !} \lambda\left(x^{\alpha}\right) \partial^{\alpha} \in \mathbb{K}[[\partial]]$. More generally, any linear form on $\langle\mathcal{P}\rangle$ can be written as $\lambda=\sum_{p \in \mathcal{P}} \lambda(p) p^{\dagger}(\partial)$.

\subsection{Least interpolation space}

For a space of linear forms $\Lambda \subset \mathbb{K}[\mathrm{x}]^{*}$, a canonical interpolation space $\Lambda_{\downarrow}$ is introduced in [5]. It has a desirable set of properties. An algorithm to build a basis of $\Lambda_{\downarrow}$ based on Gauss elimination on the Vandermonde matrix is presented in [4]. In this algorithm the authors consider the Vandermonde matrix associated to the monomial basis of $\mathbb{K}[\mathrm{x}]$. The notion of dual bases introduced above, allows to extend the algorithm to any graded basis of $\mathbb{K}[\mathrm{x}]$.

The initial term of a power series $\lambda \in \mathbb{K}[[\partial]]$, denoted by $\lambda_{\downarrow} \in$ $\mathbb{K}[\mathrm{x}]$ in [3-5], is the unique homogeneous polynomial for which $\lambda-\lambda_{\downarrow}(\partial)$ vanishes to highest possible order at the origin. Given a linear space of linear forms $\Lambda$, we define $\Lambda_{\downarrow}$ as the linear span of all $\lambda_{\downarrow}$ with $\lambda \in \Lambda$. [5, Proposition 2.10] shows that $\operatorname{dim} \Lambda=\operatorname{dim} \Lambda_{\downarrow}$.

Proposition 2.3. Let $\mathcal{P}=\left\{p_{1}, p_{2}, \ldots\right\}$ be a homogeneous basis of $\mathbb{K}[\mathrm{x}]$ ordered by degree and $\mathcal{L}=\left\{\lambda_{1}, \ldots, \lambda_{r}\right\}$ be a basis of $\Lambda$. Let $\mathrm{LU}=\mathrm{W}_{\mathcal{L}}^{\mathcal{P}}$ be the factorization of $\mathrm{W}_{\mathcal{L}}^{\mathcal{P}}$ provided by Gauss elimination with partial pivoting with $\left\{j_{1}, j_{2}, \ldots, j_{r}\right\}$ as echelon index sequence. If $\mathrm{U}=\left(u_{i j}\right)$ consider, for $1 \leq \ell \leq r$,

$$
h_{\ell}=\sum_{\operatorname{deg}\left(p_{k}\right)=\operatorname{deg}\left(p_{j_{\ell}}\right)} u_{\ell k} p_{k}^{\dagger}
$$

where $\mathcal{P}^{\dagger}=\left\{p_{1}^{\dagger}, \ldots p_{j}^{\dagger}, \ldots\right\}$ is the dual basis of $\mathcal{P}$ with respect to the apolar product. Then $H=\left\{h_{1}, \ldots h_{r}\right\}$ is a basis for $\Lambda_{\downarrow}$.

Proof. Let $\mathrm{L}^{-1}=\left(a_{i j}\right)$ and consider $\varsigma_{\ell}=\sum_{j \in \mathbb{N}} u_{\ell j} p_{j}^{\dagger}(\partial)$. Since $u_{\ell j}=\sum_{i=1}^{r} a_{l i} \lambda_{i}\left(p_{j}\right)$, then $\varsigma_{\ell}=\sum_{j \in \mathbb{N}}\left(\sum_{i=1}^{r} a_{l i} \lambda_{i}\left(p_{j}\right)\right) p_{j}^{\dagger}(\partial)$

$$
=\sum_{i=1}^{r} a_{l i} \sum_{j \in \mathbb{N}} \lambda_{i}\left(p_{j}\right) p_{j}^{\dagger}(\partial)=\sum_{i=1}^{r} a_{l i} \lambda_{i} \in \Lambda .
$$

Notice that $h_{\ell}=\varsigma_{\ell \downarrow}$ and therefore $h_{\ell} \in \Lambda_{\downarrow}$ for $1 \leq \ell \leq r$.

The $j_{i}$ are strictly increasing so that $\left\{h_{1}, h_{2}, \ldots, h_{r}\right\}$ are linearly independent. Since $\operatorname{dim}(\Lambda)=\operatorname{dim}\left(\Lambda_{\downarrow}\right)=r$ we conclude that $H$ is a basis of $\Lambda_{\downarrow}$.

\section{SYMMETRY}

We define the concepts of invariant interpolation problem (IIP) and equivariant interpolation problem (EIP). These interpolation problems have a structure that we want to be preserved by the interpolant. We show that this is automatically achieved when choosing the interpolant in an invariant interpolation space. Then the solution of an IIP is an invariant polynomial and the solution of an EIP is an equivariant polynomial map. In Section 5 we show that the least interpolation space is invariant and how to better compute an invariant interpolation space of minimal degree.
The symmetries we shall deal with are given by the linear group action of a finite group $G$ on $\mathbb{K}^{n}$. It is thus given by a representation $\vartheta$ of $G$ on $\mathbb{K}^{n}$. It induces a representation $\rho$ of $G$ on $\mathbb{K}[\mathrm{x}]$ given by

$$
\rho(g) p(x)=p\left(\vartheta\left(g^{-1}\right) x\right) .
$$

$\mathbb{K}[\mathrm{x}]_{\delta}$ is invariant under $\rho$. It also induces a linear representation on the space of linear forms, the dual representation of $\rho$ :

$$
\rho^{*}(\lambda)(p)=\lambda\left(\rho\left(g^{-1}\right) p\right), p \in \mathbb{K}[\mathrm{x}] \text { and } \lambda \in \mathbb{K}[\mathrm{x}]^{*} .
$$

We shall deal with an invariant subspace $\Lambda$ of $\mathbb{K}[\mathrm{x}]^{*}$. Hence the restriction of $\rho^{*}$ to $\Lambda$ is a linear representation of $G$ in $\Lambda$.

\subsection{Invariance}

Definition 3.1. Let $\Lambda$ be a space of linear forms and $\phi: \Lambda \longrightarrow \mathbb{K}$ a linear map. The pair $(\Lambda, \phi)$ defines an invariant interpolation problem if

(1) $\Lambda$ is closed under the action of $G$.

(2) $\phi\left(\rho^{*}(g)(\lambda)\right)=\phi(\lambda)$ for any $g \in G$ and $\lambda \in \Lambda$.

An invariant Lagrange interpolation problem can be seen as interpolation at union of orbits of points with fixed values on their orbits, i.e., given $\xi_{1}, \ldots, \xi_{m}$ with orbits $O_{1}, \ldots, O_{m}$ and $\eta_{1}, \ldots, \eta_{m} \in \mathbb{K}^{n}$, an interpolant $p \in \mathbb{K}[\mathrm{x}]$ is to satisfy $p \circ \vartheta(g)\left(\xi_{k}\right)=\eta_{k}$ for any $g \in G$. It is natural to expect that an appropriate interpolant $p$ be invariant. Yet, not all minimal degree interpolants are invariant.

Example 3.2. The dihedral group $D_{m}$ is the group of order $2 m$ that leaves invariant the regular $m$-gon. It thus has a representation in $\mathbb{R}^{2}$ given by the matrices

$\vartheta_{k}=\left[\left(\begin{array}{cc}\cos \left(\left\lfloor\frac{k}{2}\right\rfloor \frac{2 \pi}{m}\right) & -\sin \left(\left\lfloor\frac{k}{2}\right\rfloor \frac{2 \pi}{m}\right) \\ \sin \left(\left\lfloor\frac{k}{2}\right\rfloor \frac{2 \pi}{m}\right) & \cos \left(\left\lfloor\frac{k}{2}\right\rfloor \frac{2 \pi}{m}\right)\end{array}\right)\left(\begin{array}{cc}1 & 0 \\ 0 & -1\end{array}\right)^{k}\right], 0 \leq k \leq 2 m-1$.

Consider $\Xi \subset \mathbb{R}^{2}$ a set of $1+3 \times 5$ point illustrated on Figure 1 . They form four orbits $O_{1}, O_{2}, O_{3}, O_{4}$ of $D_{5}$ so that $\Lambda:=\operatorname{span}\left(\mathbb{e}_{\xi_{i}} \mid \xi_{i} \in \Xi\right)$ is invariant. An invariant interpolation problem is given by the pair $(\Lambda, \phi)$ where $\phi$ is defined by $\phi\left(\mathbb{e}_{\xi}\right)=0.1$ if $\xi \in O_{1}, \phi\left(\mathbb{e}_{\xi}\right)=0$ if $\xi \in O_{2} \cup O_{4}$, and $\phi\left(\mathbb{e}_{\xi}\right)=-0.5$ if $\xi \in O_{3}$. We show in Figure 1 the graph of the expected interpolant, but in Figure 2 we present the graph of an interpolant of minimal degree.

Proposition 3.3. Let $(\Lambda, \phi)$ be an invariant interpolation problem. Let $P$ be an invariant interpolation space and let $p \in \mathbb{K}[\mathrm{x}]$ be the solution of $(\Lambda, \phi)$ in $P$. Then $p \in \mathbb{K}[\mathrm{x}]^{G}$, the ring of invariant polynomials.

Proof. For any $\lambda \in \Lambda$ and $g \in G$ we have that $\lambda(p)=\phi(\lambda)$ and $\rho^{*}(g)(\lambda) p=\phi\left(\rho^{*}(g)(\lambda)\right)$. Since $\phi$ is $G$-invariant, we get that $\lambda\left(\rho\left(g^{-1}\right) p\right)=\rho^{*}(g)(\lambda) p=\phi\left(\rho^{*}(g)(\lambda)\right)=\lambda(p)$ for any $\lambda \in \Lambda$. The latter implies that $\rho\left(g^{-1}\right) p-p \in \operatorname{Ker} \Lambda$. As $P$ is closed under the action of $\rho, \rho\left(g^{-1}\right) p-p \in \operatorname{Ker} \Lambda \cap P$. Then as $(\Lambda, P)$ is an interpolation space $\operatorname{Ker} \Lambda \cap P=\emptyset$ and we conclude that $\rho\left(g^{-1}\right) p-$ $p=0$ for any $g \in G$, i.e., $p \in \mathbb{K}[\mathrm{x}]^{G}$. 


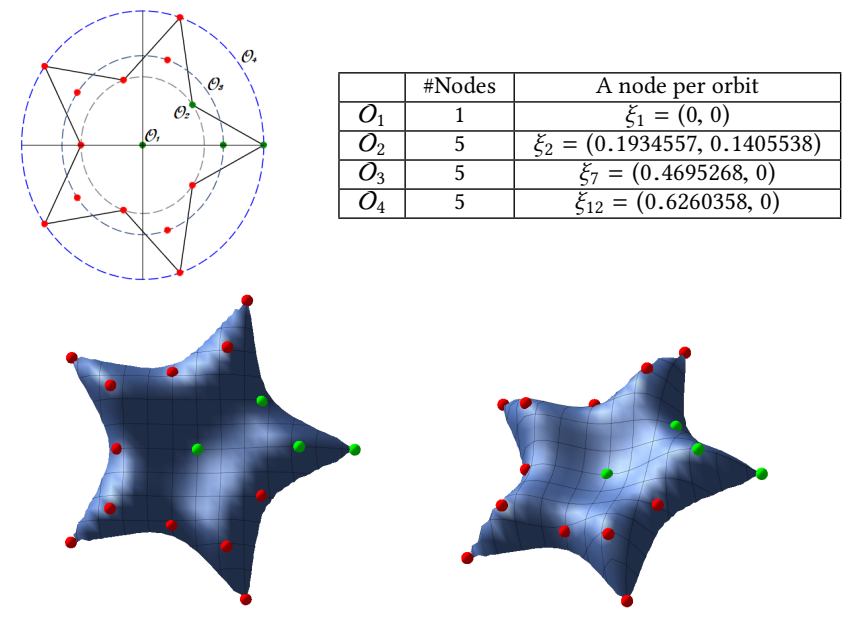

Figure 1: Invariant Lagrange interpolation problem and invariant interpolant of minimal degree.
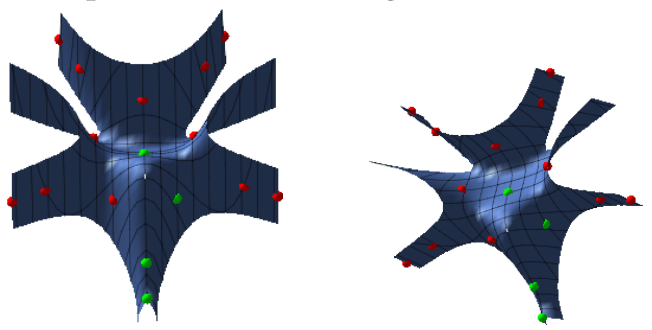

Figure 2: Graph of a minimal degree interpolant obtained from a monomial basis. The $D_{5}$ symmetry is not respected.

\subsection{Equivariance}

Let $\mathbb{K}[\mathrm{x}]^{m}$ be the module of polynomial mappings with $m$ components, and let $\theta: G \longrightarrow \operatorname{Aut}\left(\mathbb{K}^{m}\right)$ be a linear representation on $\mathbb{K}^{m}$. A polynomial mapping $f=\left(f_{1}, f_{2}, \ldots, f_{m}\right)^{t}$ is called $\vartheta-\theta$ equivariant if $f(\vartheta(g) x)=\theta(g) f(x)$ for any $g \in G$. The space of equivariant mappings over $\mathbb{K}$, denoted by $\mathbb{K}[\mathrm{x}]_{\vartheta}^{\theta}$, is a $\mathbb{K}[\mathrm{x}]^{G}$-module.

Equivariant maps define, for instance, dynamical systems that exhibit particularly interesting patterns and are relevant to model physical or biological phenomena $[1,12]$. In this context, it is interesting to have a tool to offer equivariant maps that interpolate some observed local behaviors.

Definition 3.4. Let $\Lambda$ be a space of linear forms on $\mathbb{K}[\mathrm{x}]$ and $\phi$ : $\Lambda \longrightarrow \mathbb{K}^{m}$ a linear map. The pair $(\Lambda, \phi)$ defines a $\vartheta-\theta$ equivariant interpolation problem if

(1) $\Lambda$ is closed under the action of $G$.

(2) $\phi\left(\rho^{*}\left(g^{-1}\right)(\lambda)\right)=\theta(g) \phi(\lambda)$ for any $g \in G$ and $\lambda \in \Lambda$.

The solution of an $\operatorname{EIP}(\Lambda, \phi)$, is a polynomial map $f=\left(f_{1}, \ldots, f_{m}\right)^{t}$ such that $\lambda(f)=\left(\lambda\left(f_{1}\right), \ldots, \lambda\left(f_{m}\right)\right)^{t}=\phi(\lambda)$ for any $\lambda \in \Lambda$. It is natural to seek $f$ as an equivariant map. It is remarkable that any type of equivariance will be respected as soon as the interpolation space is invariant.

Proposition 3.5. Let $(\Lambda, \phi)$ be an equivariant interpolation problem. Let $P$ be an invariant interpolation space for $\Lambda$ and let $f=$ $\left(f_{1}, \ldots, f_{m}\right)^{t}$ be the solution of $(\Lambda, \phi)$ in $P$. Then $f \in \mathbb{K}[x]_{\vartheta}^{\theta}$.
Proof. For any $\lambda \in \Lambda$ we have the following

$$
\rho^{*}(g)(\lambda) f=\phi\left(\rho^{*}(g) \lambda\right)=\theta(g) \phi(\lambda)=\theta(g) \lambda(f)=\lambda(\theta(g) f) .
$$

We can write $\theta(g) f$ as $\left(\sum_{i=1}^{m} r_{1 i} f_{i}, \ldots, \sum_{i=1}^{m} r_{m i} f_{i}\right)$, where $\left(r_{i j}\right)$ is a matrix representation of $\theta(g)$. By equation (5) we get

$$
\left(\lambda\left(\rho\left(g^{-1}\right) f_{1}\right), \ldots, \lambda\left(\rho\left(g^{-1}\right) f_{m}\right)\right)=\left(\lambda\left(\sum_{i=1}^{m} r_{1 i} f_{i}\right), \ldots, \lambda\left(\sum_{i=1}^{m} r_{m i} f_{i}\right)\right),
$$

and therefore $\rho\left(g^{-1}\right) f_{j}-\sum_{i=1}^{m} r_{j i} f_{i} \in \operatorname{Ker} \Lambda \bigcap P=\emptyset$ for any $1 \leq j \leq$ $m$ which implies that $\left(f_{1} \circ \vartheta\left(g^{-1}\right), \ldots, f_{m} \circ \vartheta\left(g^{-1}\right)\right)=\theta(g) f$.

Example 3.6. The symmetry is given by the representation of the dihedral group $D_{3}$ in Equation (4). The space $\Lambda$ of linear forms we consider is spanned by the evaluations at the points of the orbits $O_{1}$ and $O_{2}$ of $\xi_{1}=\left(-\frac{5 \sqrt{3}}{3}, \frac{1}{3}\right)^{t}$ and $\xi_{2}=\left(-\sqrt{3}, \frac{1}{3}\right)^{t}$. We define $\phi: \Lambda \rightarrow \mathbb{K}^{2}$ by

$$
\phi\left(\mathbb{e}_{\vartheta}(g) \xi_{1}\right)=\vartheta(g)\left(\begin{array}{l}
a \\
c
\end{array}\right) \quad \text { and } \quad \phi\left(\mathbb{e}_{\vartheta}(g) \xi_{2}\right)=\vartheta(g)\left(\begin{array}{l}
b \\
d
\end{array}\right) .
$$

The thus defined interpolation problem is clearly $\vartheta-\vartheta$ equivariant. For each quadruplet $(a, b, c, d) \in \mathbb{K}^{4}$ it is desirable to find an interpolant $\left(p_{1}, p_{2}\right)^{t} \in \mathbb{K}[x]^{2}$ that is an $\vartheta-\vartheta$ equivariant map. This will define the equivariant dynamical system

$$
\dot{x_{1}}(t)=p_{1}\left(x_{1}(t), x_{2}(t)\right), \quad \dot{x_{2}}(t)=p_{2}\left(x_{1}(t), x_{2}(t)\right)
$$

whose integral curves, limit cycles and equilibrium points, will all exhibit the $D_{3}$ symmetry. In Figure 3 we draw the integral cuves of equivariant vector field thus constructed. The data of the interpolation problem are illustrated by the black arrows : they are the vectors $(a, c)^{t}$ and $(b, d)^{t}$, with origin in the points $\xi_{1}$ and $\xi_{2}$, together with their transforms.

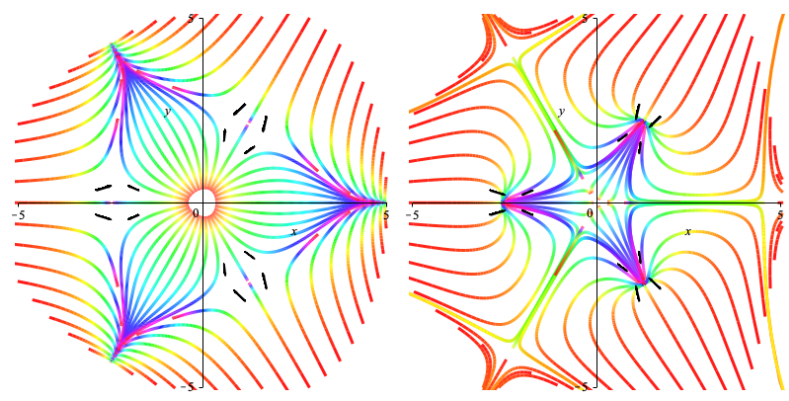

Figure 3: Integral curves for the equivariant vector field interpolating the invariant set of 12 vectors drawn in black

\section{SYMMETRY REDUCTION}

In this section we show how, when the space $\Lambda$ of linear forms is invariant, the Vandermonde matrix can be made block diagonal. That happens when making use of symmetry adapted bases both for $\mathbb{K}[\mathrm{x}]_{\leq \delta}$ and $\Lambda$. We start by recalling their general construction, as it appears in representation theory. The material is drawn from $[6,21]$. This block diagonalisation of the Vandermonde indicates how computation can be organized more efficiently, and robustly. It 
just draws on the invariance of the space of linear forms. So, when the evaluation points can be chosen, it makes sense to introduce symmetry among them.

\subsection{Symmetry adapted bases}

A linear representation of the group $G$ on the $\mathbb{C}$-vector space $V$ is a group morphism from $G$ to the group $G L(V)$ of isomorphisms from $V$ to itself. $V$ is called the representation space and $n$ is the dimension (or the degree) of the representation $\rho$. If $V$ has finite dimension $n$, and $\rho$ is a linear representation of $G$ on $V$, upon introducing a basis $\mathcal{P}$ of $V$ the isomorphism $\rho(g)$ can be described by a non-singular $n \times n$ matrix. This representing matrix is denoted by $[\rho(g)] \mathcal{\rho}$. The complex-value function $\chi: G \longrightarrow \mathbb{C}$, with $\chi(g) \rightarrow \operatorname{Trace}(\rho(g))$ is the character of the representation $\rho$.

The dual or contragredient representation of $\rho$ is the representation $\rho^{*}$ on the dual vector space $V^{*}$ defined by:

$$
\rho^{*}(g)(\lambda)=\lambda \circ \rho\left(g^{-1}\right) \text { for any } \lambda \in V^{*} .
$$

If $\mathcal{P}$ is a basis of $V$ and $\mathcal{P}^{*}$ its dual basis then $\left[\rho^{*}(g)\right] \mathcal{P}^{*}=\left[\rho\left(g^{-1}\right)\right]_{\mathcal{P}}^{t}$. It follows that $\chi_{\rho^{*}}(g)=\chi_{\rho}\left(g^{-1}\right)=\bar{\chi}_{\rho}(g)$

A linear representation $\rho$ of a group $G$ on a space $V$ is irreducible if there is no proper subspace $W$ of $V$ with the property that, for every $g \in G$, the isomorphism $\rho(g)$ maps every vector of $W$ into $W$. In this case, its representation space $V$ is also called irreducible. The contragredient representation $\rho^{*}$ is irreducible when $\rho$ is. A finite group has a finite number of inequivalent irreducible representations. Any representation of a finite group is completely reducible, meaning that it decomposes into a finite number of irreducible subspaces.

Let $\rho_{j}(j=1, \ldots, N)$ be the irreducible $n_{j}$ dimensional representations of $G$. The complete reduction of the representation $\rho$ and its representation space are denoted by $\rho=c_{1} \rho_{1} \oplus \cdots \oplus c_{N} \rho_{N}$ and $V=$ $V_{1} \oplus \cdots \oplus V_{N}$. Each invariant subspace $V_{j}$ is the direct sum of $c_{j}$ irreducible subspaces and the restriction of $\rho$ to each one is equivalent to $\rho_{j}$. The $\left(c_{j} n_{j}\right)$-dimensional subspaces $V_{j}$ of $V$ are the isotypic components. With $\chi_{j}$ the character of $\rho_{j}$ we determine the multiplicity $c_{j}$ and the projection $\pi_{j}$ onto the isotypic component $V_{j}$

$$
c_{j}=\frac{1}{|G|} \sum_{g \in G} \overline{\chi_{j}(g)} \chi(g), \quad \pi_{j}=\frac{n_{j}}{|G|} \sum_{g \in G} \chi_{j}\left(g^{-1}\right) \rho(g) .
$$

To go further in the decomposition, consider the representing matrices $\mathrm{R}_{j}(g)=\left(r_{\alpha \beta}^{j}(g)\right)_{1 \leq \alpha, \beta \leq n_{j}}$ for $\rho_{j}$. For $1 \leq \alpha, \beta \leq n_{j}$, let

$$
\pi_{j, \alpha \beta}=\frac{n_{j}}{|G|} \sum_{g \in G} r_{\beta \alpha}^{j}\left(g^{-1}\right) \rho(g) .
$$

Let $\left\{p_{1}^{j}, \ldots, p_{c_{j}}^{j}\right\}$ be a basis of the subspace $V_{j, 1}=\pi_{j, 11}(V)$. A symmetry adapted basis of the isotypic component $V_{j}$ is then given by

$$
\mathcal{P}_{j}=\left\{p_{1}^{j}, \ldots, p_{c_{j}}^{j}, \ldots, \pi_{j, n_{j} 1}\left(p_{1}^{j}\right), \ldots, \pi_{j, n_{j} 1}\left(p_{c_{j}}^{j}\right)\right\} .
$$

The union $\mathcal{P}$ of the $\mathcal{P}_{j}$ of $V_{j}$, is a symmetry adapted basis for $V$. Indeed, by [21, Proposition 8], the set $\left\{\pi_{j, \alpha 1}\left(p_{1}^{j}\right), \ldots, \pi_{j, \alpha 1}\left(p_{c_{j}}^{j}\right)\right\}$ is a basis of $V_{j, \alpha}=\pi_{j, \alpha \alpha}(V)$ and $V_{j}=V_{j, 1} \oplus \cdots \oplus V_{j, n_{j}}$. Furthermore $\left\{p_{k}^{j}, \pi_{j, 21}\left(p_{k}^{j}\right), \ldots, \pi_{j, n_{j} 1}\left(p_{k}^{j}\right)\right\}$ is a basis of an irreducible space with representating matrices $\left(r_{\alpha \beta}^{j}(g)\right)_{1 \leq \alpha, \beta \leq n_{j}}$. Hereafter we denote by $\mathcal{P}^{j, \alpha}$ the polynomial map defined by

$$
\mathcal{P}^{j, \alpha}=\left(\pi_{j, \alpha 1}\left(p_{1}^{j}\right), \ldots, \pi_{j, \alpha 1}\left(p_{c_{j}}^{j}\right)\right) .
$$

A symmetry adapted basis $\mathcal{P}$ is characterized by the fact that $[\rho(g)]_{\mathcal{P}}=\operatorname{diag}\left(\mathrm{R}_{1}(g) \otimes \mathrm{I}_{c_{1}}, \ldots, \mathrm{R}_{N}(g) \otimes \mathrm{I}_{c_{N}}\right)$.Then $\left[\rho^{*}(g)\right]_{\mathcal{P}^{*}}=$ $\operatorname{diag}\left(\mathrm{R}_{i}^{-t}(g) \otimes \mathrm{I}_{c_{i}} \mid i=1 . . N\right)$.

Proposition 4.1. If $\mathcal{P}=\cup_{i=1}^{N} \mathcal{P}_{i}$ be a symmetry adapted basis of $V$ where $\mathcal{P}_{i}$ spans the isotypic component associated to $\rho_{i}$ then its dual basis $\mathcal{P}^{*}=\cup_{i=1}^{N} \mathcal{P}_{i}^{*}$ in $V^{*}$ is a symmetry adapted basis where $\mathcal{P}_{i}^{*}$ spans the isotypic component associated to $\rho_{i}^{*}$.

CoROLlary 4.2. If $\mathcal{P}$ is a symmetry adapted basis of $\mathbb{K}[\mathrm{x}]_{\leq \delta}$, so is its dual $\mathcal{P}^{\dagger}$ with respect to the apolar product.

A scalar product is $G$-invariant with respect to a linear representation $\rho$ if $\langle v, w\rangle=\langle\rho(g)(v), \rho(g)(w)\rangle$ for any $g \in G$ and $v, w \in V$. If we consider unitary representing matrices $\mathrm{R}_{i}(g)$, and an orthonormal basis $\left\{p_{1}^{j}, \ldots, p_{c_{j}}^{j}\right\}$ of $V_{i, 1}$ with respect to a $G$-invariant inner product, then the same process leads to an orthonormal symmetry adapted basis [6, Theorem 5.4].

Some irreducible representations might not have representing matrices in $\mathbb{R}$. Yet one can determine a real symmetry adapted basis [2] by combining the isotypic components related to conjugate irreducible representations. This happens for instance for abelian groups and we shall avoid them in the examples of this paper for lack of space. Indeed the completely general statements become convoluted when working with the distinction.

\subsection{Block diagonal Vandermonde matrix}

We consider a linear representation $\vartheta$ of a finite group $G$ on $\mathbb{K}^{n}$. It induces the representations $\rho$ and its dual $\rho^{*}$ on the space $\mathbb{K}[\mathrm{x}]$ and $\mathbb{K}[\mathrm{x}]^{*} . \mathbb{K}[\mathrm{x}]_{\delta}$ is invariant under $\rho$ and thus can be decomposed into isotypic components $\mathbb{K}[\mathrm{x}]_{\delta}=\bigoplus_{j=1}^{N} P_{j}$, where $P_{j}$ is associated to the irreducible representation $\rho_{j}$ of $G$, with character $\chi_{j}$. Each $P_{j}$ is the image of $\mathbb{K}[\mathrm{x}]_{\delta}$ under the map $\pi_{j}$, as defined in (7).

For an invariant subspace $\Lambda$ of $\mathbb{K}[\mathrm{x}]^{*}$ the restriction of $\rho^{*}$ to $\Lambda$ is a linear representation of $G$. We shall arrange the isotypic decomposition $\Lambda=\Lambda_{1}^{*} \oplus \ldots \oplus \Lambda_{N}^{*}$ such that $\Lambda_{j}^{*}$ is the isotypic component associated to the irreducible representation $\rho_{j}^{*}$, with character $\overline{\chi_{j}}$. To make a distinction we denote $\pi_{j, \alpha \beta}^{*}$ as the map defined in (8) associated to $\rho^{*}$.

Proposition 4.3. Let $\rho$ and $\rho^{*}$ be linear representations of a finite group $G$ on $\mathbb{K}[\mathrm{x}]_{\leq \delta}$ and $\Lambda$ defined as above. Let $\mathcal{P}=\bigcup_{j=1}^{N} \mathcal{P}^{j}$ be a symmetry adapted basis of $\mathbb{K}[\mathrm{x}]_{\leq \delta}$ with

- $\left\{p_{1}^{j}, \ldots, p_{c_{j}}^{j}\right\}$ a basis of $\pi_{j, 11}\left(\mathbb{K}[\mathrm{x}]_{\leq \delta}\right)$.

- $\mathcal{P}^{j}=\left\{p_{1}^{j}, \ldots, p_{c_{j}}^{j}, \ldots, \pi_{j, n_{j} 1}\left(p_{1}^{j}\right), \ldots, \pi_{j, n_{j} 1}\left(p_{c_{j}}^{j}\right)\right\}$.

Let $\mathcal{L}=\bigcup_{j=1}^{N} \mathcal{L}^{j}$ be a symmetry adapted basis of $\Lambda$ with

- $\left\{\lambda_{1}^{j}, \ldots, \lambda_{r_{j}}^{j}\right\}$ a basis of $\pi_{j, 11}^{*}(\Lambda)$.

- $\mathcal{L}^{j}=\left\{\lambda_{1}^{j}, \ldots, \lambda_{r_{j}}^{j}, \ldots, \pi_{j, n_{j} 1}^{*}\left(\lambda_{1}^{j}\right), \ldots, \pi_{j, n_{j} 1}^{*}\left(\lambda_{r_{j}}^{j}\right)\right\}$. 
Then the Vandermonde matrix $\mathrm{W}_{\mathcal{L}}^{\mathcal{P}}$ is given by

$$
\operatorname{diag}\left(\mathrm{I}_{n_{j}} \otimes\left(\lambda_{s}^{j}\left(p_{t}^{j}\right)\right)_{\substack{1 \leq s \leq r_{j} \\ 1 \leq t \leq c_{j}}}, i=1 \ldots N\right),
$$

where $\otimes$ denotes the Kronecker product.

Proof. Let $\alpha, \beta, \gamma, \sigma \in \mathbb{N}$ such that $1 \leq \alpha, \beta \leq n_{j}$ and $1 \leq$ $\gamma, \sigma \leq n_{i}$. Let $\lambda_{\alpha \beta}^{j}=\pi_{j, \alpha 1}^{*}\left(\lambda_{\beta}^{j}\right)$ and $p_{\gamma \sigma}^{i}=\pi_{i, \gamma 1}\left(p_{\sigma}^{i}\right)$. For any entry $\lambda_{\alpha \beta}^{j}\left(p_{\gamma \sigma}^{i}\right)$ in $\mathrm{W}_{\mathcal{L}}^{\mathcal{P}}$ we have the following:

$$
\begin{gathered}
\lambda_{\alpha \beta}^{j}\left(p_{\gamma \sigma}^{i}\right)=\lambda_{\alpha \beta}^{j}\left(\pi_{i, \gamma 1}\left(p_{\sigma}^{i}\right)\right)=\lambda_{\alpha \beta}^{j}\left(\frac{n_{i}}{|G|} \sum_{g \in G} r_{1 \gamma}^{i}\left(g^{-1}\right) \rho(g)\left(p_{\sigma}^{i}\right)\right) \\
=\frac{n_{i}}{|G|} \sum_{g \in G} r_{1 \gamma}^{i}\left(g^{-1}\right) \rho^{*}\left(g^{-1}\right)\left(\lambda_{\alpha \beta}^{j}\right)\left(p_{\sigma}^{i}\right) \\
=\frac{n_{i}}{|G|} \sum_{g \in G} r_{\gamma 1}^{i}(g) \rho^{*}\left(g^{-1}\right)\left(\lambda_{\alpha \beta}^{j}\right)\left(p_{\sigma}^{i}\right)=\pi_{i, 1 \gamma}^{*}\left(\lambda_{\alpha \beta}^{j}\right)\left(p_{\sigma}^{i}\right) .
\end{gathered}
$$

Using Proposition [21, Proposition 8] (2) if $i \neq j, \pi_{i, 1 \gamma}^{*}\left(\lambda_{\alpha \beta}^{j}\right)=0$ then $\lambda_{\alpha \beta}^{j}\left(p_{\gamma \sigma}^{i}\right)$ is zero for $i \neq j$, i.e., $\mathrm{W}_{\mathcal{L}}^{\mathcal{P}}$ is block diagonal in the isotypic components. Now if $i=j$

$$
\lambda_{\alpha \beta}^{j}\left(p_{\gamma \sigma}^{j}\right)=\pi_{j, 1 \gamma}^{*}\left(\lambda_{\alpha \beta}^{j}\right)\left(p_{\sigma}^{j}\right)=\pi_{j, 1 \gamma}^{*} \circ \pi_{j, \alpha 1}^{*}\left(\lambda_{\beta}^{j}\right)\left(p_{\sigma}^{j}\right) .
$$

Since $\pi_{j, 1 \gamma}^{*} \circ \pi_{j, \alpha 1}^{*}\left(\lambda_{\beta}^{j}\right)\left(p_{\sigma}^{j}\right)=\left\{\begin{array}{c}\pi_{j, 11}^{*}\left(\lambda_{\beta}^{j}\right)\left(p_{\sigma}^{j}\right) \text { if } \alpha=\gamma \\ 0 \text { otherwise }\end{array}\right.$, using the fact that $\pi_{j, 11}^{*}\left(\lambda_{\beta}^{j}\right)=\lambda_{\beta}^{j}$ we get that

$$
\lambda_{\alpha \beta}^{j}\left(p_{\gamma \sigma}^{i}\right)=\left\{\begin{array}{cl}
\lambda_{\beta}^{j}\left(p_{\sigma}^{j}\right) \text { if } i=j \text { and } \alpha=\gamma \\
0 & \text { otherwise. }
\end{array}\right.
$$

Thus the Vandermonde matrix $\mathrm{W}_{\mathcal{L}}^{\mathcal{P}}$ has the announced block diagonal structure.

REMARK 1. At the heart of the above proof is the following property : for a representation $\mathcal{V}=\bigoplus_{j=1}^{N} \mathcal{V}_{i}$ of $G$, and its dual $\mathcal{V}^{*}=$ $\bigoplus_{j=1}^{N} \mathcal{V}_{i}^{*}$, we have $\lambda(v)=0$ as soon as $\lambda \in \mathcal{V}_{i}^{*}$ while $v \in \mathcal{V}_{j}$ for $i \neq j$.

Example 4.4. Let $G$ be the dihedral group $D_{3}$ of order 6. A representation of $G$ on $\mathbb{R}^{2}$ is given by Equation (4) with $m=3$. $D_{3}$ has three irreducible representations, two of dimension 1 and one of dimension 2.

Consider $\Xi$ the orbit of the point $\xi_{1}=\left(-\frac{5 \sqrt{3}}{3}, \frac{1}{3}\right)^{t}$ in $\mathbb{R}^{2}$, with $\xi_{i}=\vartheta_{i-1} \xi_{1}$. Let $\Lambda=\operatorname{span}\left(\mathbb{E}_{\xi_{i}} \circ D_{\vec{\xi}_{i}}\right)$ with $D_{\vec{\xi}_{i}}$ the directional derivative with direction $\vec{\xi}_{i} . \Lambda$ is closed under the action of $G$. Indeed for any $p \in \mathbb{K}[\mathrm{x}], \rho^{*}(g)\left(\mathbb{e}_{\xi_{i}} \circ D_{\vec{\xi}_{i}}\right)(p)=\mathbb{e}_{\xi_{i}} \circ D_{\vec{\xi}_{i}}\left(p\left(\vartheta\left(g^{-1} x\right)\right)=\right.$ $\mathbb{e}_{\vartheta\left(g^{-1}\right) \xi_{i}} \circ D_{\vartheta\left(g^{-1}\right) \xi_{i}}(p(x))$. Since $\vartheta\left(g^{-1}\right) \xi_{i}=\xi_{j}$ for some $1 \leq j \leq 6$ we have $\rho^{*}(g)\left(\mathbb{E}_{\xi_{i}} \circ D_{\vec{\xi}_{i}}\right)=\mathbb{e}_{\xi_{j}} \circ D_{\vec{\xi}_{j}}$. Considering $\varrho_{i}=\mathbb{e}_{\xi_{i}} \circ D_{\vec{\xi}_{i}}$ a symmetry adapted basis of $\Lambda$ is given by

$$
\mathcal{L}:=\left[\begin{array}{c}
{\left[\varrho_{1}+\varrho_{2}+\varrho_{3}+\varrho_{4}+\varrho_{5}+\varrho_{6}\right]} \\
{\left[\varrho_{1}-\varrho_{2}+\varrho_{3}-\varrho_{4}+\varrho_{5}-\varrho_{6}\right]} \\
{\left[\left[\lambda_{3}, \lambda_{4}\right],\left[\lambda_{5}, \lambda_{6}\right]\right]}
\end{array}\right]
$$

with $\lambda_{3}=\varrho_{1}+\varrho_{2}-\varrho_{4}-\varrho_{5} \quad \lambda_{5}=\frac{\sqrt{3}}{2}\left(\varrho_{2}-\varrho_{1}+\varrho_{4}+2 \varrho_{3}-2 \varrho_{6}-\varrho_{5}\right)$, $\lambda_{4}=\varrho_{3}-\varrho_{4}-\varrho_{5}+\varrho_{6}, \quad \lambda_{6}=\frac{\sqrt{3}}{2}\left(2 \varrho_{2}-2 \varrho_{1}-\varrho_{4}+\varrho_{3}-\varrho_{5}-\varrho_{6}\right)$. A symmetry adapted basis of $\mathbb{R}[x]_{\leq 3}$ is given by

$$
\mathcal{P}:=\left[\begin{array}{c}
{\left[1, x_{1}^{2}+x_{2}^{2}, x_{1}^{3}-3 x_{1} x_{2}^{2}\right]} \\
{\left[x_{1}^{2} x_{2}-\frac{1}{3} x_{2}^{3}\right]} \\
{\left[\left[x_{1}, x_{1}^{2}-x_{2}^{2}, x_{1}^{3}+x_{1} x_{2}^{2}\right],\left[x_{2},-2 x_{1} x_{2}, x_{1}^{2} x_{2}+x_{2}^{3}\right]\right]}
\end{array}\right] .
$$

The Vandermonde matrix $\mathrm{W}_{\mathcal{L}}^{\mathcal{P}}$ is block diagonal :

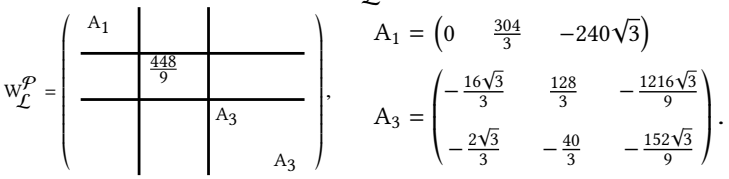

\section{EQUIVARIANT INTERPOLATION}

In this section we shall first show how to build interpolation spaces of minimal degree that are invariant. We shall actually build symmetry adapted bases for these, exploiting the block diagonal structure of the Vandermonde matrix. Doing so we prove that the least interpolation space is invariant. We then present a selection of invariant or equivariant interpolation problems. As proved in Section 3, the invariance or equivariance is preserved by the interpolant when the interpolation space is invariant. The use of the symmetry adapted bases constructed allows this equivariance to be preserved exactly, independently of the numerical accuracy.

\subsection{Constructing invariant interpolation spaces}

The starting point is a representation $\vartheta$ of $G$ on $\mathbb{K}^{n}$ that induces representations $\rho$ and $\rho^{*}$ on $\mathbb{K}[\mathrm{x}]$ and $\mathbb{K}[\mathrm{x}]^{*}$. It is no loss of generality to assume that $\vartheta$ is an orthogonal representation. The apolar product is thus $G$-invariant.

Let $\Lambda$ be an invariant subspace of $\mathbb{K}[\mathrm{x}]^{*}$. Hereafter $\mathcal{L}$ is a symmetry adapted basis of $\Lambda$ and $\mathcal{P}$ a symmetry adapted basis of $\mathbb{K}[\mathrm{x}]_{\leq \delta}$ consisting of homogeneous polynomials. The elements of $\mathcal{P}$ corresponding to the same irreducible component are ordered by degree.

According to Proposition 4.3, $\mathrm{W}_{\mathcal{L}}^{\mathcal{P}}=\operatorname{diag}\left(I_{n_{i}} \otimes \mathrm{A}_{i}\right)$. In the factorization $\mathrm{L}_{i} \mathrm{U}_{i}:=\mathrm{A}_{i}$ provided by Gauss elimination, let $j_{1}, j_{2}, \ldots, j_{r_{j}}$ be the echelon index sequence of $\mathrm{U}_{i} ; r_{i}$ is the multiplicity of $\rho_{i}^{*}$ in $\Lambda$. An echelon index sequence for $\mathrm{D}_{i}=I_{n_{i}} \otimes \mathrm{A}_{i}$ is given by

$$
S_{i}=\bigcup_{k=0}^{c_{i}-1}\left\{j_{1}+k n_{i}, j_{2}+k n_{i}, \ldots, j_{r_{i}}+k n_{i}\right\}
$$

An echelon index sequence of $\mathrm{W}_{\mathcal{L}}^{\mathcal{P}}$ is given by $S=\bigcup_{i=1}^{N} S_{i}$. Let $\mathcal{P}_{\Lambda}^{i}$ be the set of elements of $\mathcal{P}^{i}$ that are indexed by elements of $S_{i}$. From (9) we get that

$$
\mathcal{P}_{\Lambda}^{i}=\left\{b_{j_{1}}^{i}, \ldots, b_{j_{r_{i}}}^{i}, \ldots, \pi_{i, n_{i} 1}\left(b_{j_{1}}^{i}\right), \ldots, \pi_{i, n_{i} 1}\left(b_{j_{r_{i}}}^{i}\right)\right\}
$$

We prove the assertions made on the outputs of the algorithm.

Proposition 5.1. The set of polynomials $\mathcal{P}_{\Lambda}$ built it in Algorithm 1 spans a minimal degree interpolation space for $\Lambda$ that is invariant under the action of $\rho . \mathcal{P}_{\Lambda}$ is furthermore a symmetry adapted basis for this space. 


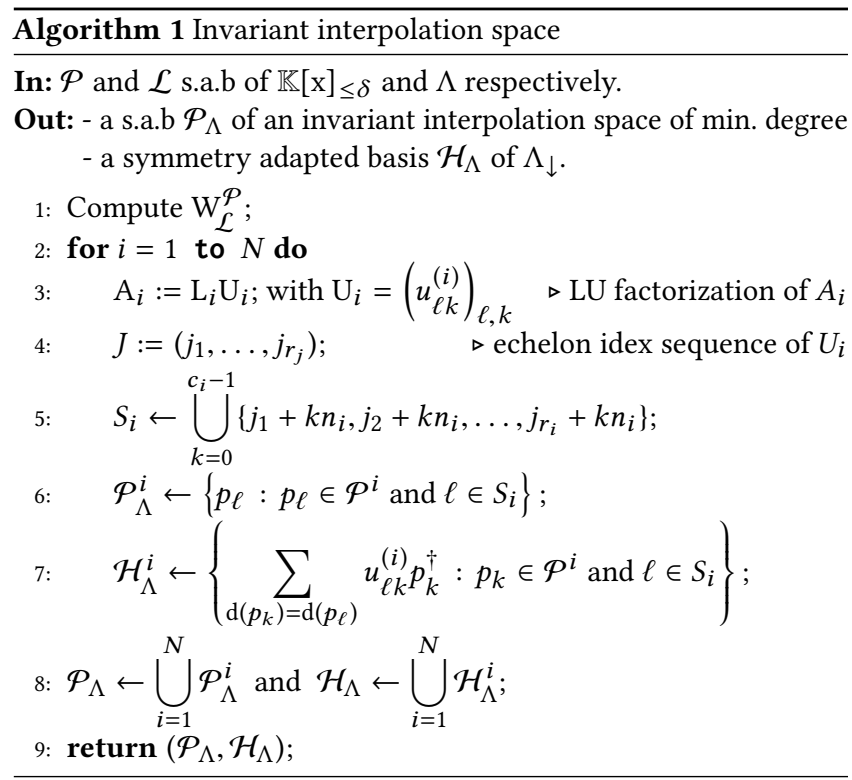

Proof. Since the elements of $P_{\Lambda}$ are indexed by the elements of $S$ then $\mathrm{W}_{\mathcal{L}}^{\mathcal{P}_{\Lambda}}$ is invertible and therefore $P_{\Lambda}$ is an interpolation space for $\Lambda$. The elements of $\mathcal{P}_{\Lambda}$ that correspond to the same blocks of $\mathrm{W}_{\mathcal{L}}^{\mathcal{P}}$ are ordered by degree. Then as a direct consequence of Proposition 2.2, $P_{\Lambda}$ is a minimal degree interpolation space. We prove now that for any $p$ in $\mathcal{P}_{\Lambda}, \rho(g)(p) \in P_{\Lambda}$. Considering $p=\pi_{j, \alpha 1}(b)$. By Proposition [21, Proposition 8] (3) we have that $\rho(g)(p)=\sum_{\beta=1}^{n_{j}} r_{\beta \alpha}^{j}(g) \pi_{j, \beta 1}(b)$. As $\pi_{j, \beta 1}(b) \in P_{\Lambda}$ for any $1 \leq \beta \leq n_{j}$ we conclude that $\rho(g)(p) \in P_{\Lambda}$. Since $\mathcal{P}_{\Lambda}$ is a basis of $P_{\Lambda}$ we can conclude that $P_{\Lambda}$ is invariant under the action of $\rho$.

Proposition 5.2. The set $\mathcal{H}_{\Lambda}$ built it in Algorithm 1 is a symmetry adapted basis for $\Lambda_{\downarrow}$.

Proof. By Proposition 2.3 we get that $\mathcal{H}_{\Lambda}$ is a basis of $\Lambda_{\downarrow}$. Let $\mathcal{H}_{j}^{\alpha}=\left\{h_{1, \alpha}^{j}, \ldots, h_{m_{j}, \alpha}^{j}\right\}=V_{j}^{\alpha} \cap \mathcal{H}_{\Lambda}$ with $1 \leq \alpha \leq c_{j}$. By the block diagonal structure and Corollary 4.2 we have

$$
h_{\ell, \alpha}^{j}=\sum_{k} u_{\ell k}^{(j)} \pi_{j, \alpha 1}\left(q_{k}^{j}\right)=\pi_{j, \alpha 1}\left(\sum_{k} u_{\ell k}^{(j)} q_{k}^{j}\right)=\pi_{j, \alpha 1}\left(h_{\ell, 1}^{j}\right) .
$$

Therefore $\mathcal{H}_{\Lambda}^{j}$ has the following structure

$$
\mathcal{H}_{\Lambda}^{j}=\left\{h_{1,1}^{j}, \ldots, h_{m_{j}, 1}^{j}, \ldots, \pi_{j, n_{j} 1}\left(h_{1,1}^{j}\right), \ldots, \pi_{j, n_{j} 1}\left(h_{m_{j}, 1}^{j}\right)\right\} .
$$

Since for any $\ell, h_{\ell}, \pi_{j, 21}\left(h_{\ell}\right), \ldots \pi_{j, n_{j} 1}\left(h_{\ell}\right)$ form a basis of an irreducible representation of $G$ we can conclude that $\mathcal{H}_{\Lambda}$ is a symmetry adapted basis of $\Lambda$.

As pointed out in Section 4.1, we can construct a symmetry adapted basis $\mathcal{P}$ of $\mathbb{K}[\mathrm{x}]_{\delta}$ that is orthonormal with respect to the apolar product. Then $\mathcal{P}=\mathcal{P}^{\dagger}$ and the basis $\mathcal{P}_{\Lambda}$ built in Algorithm 1 is orthonormal. Moreover if in the third step of Algorithm 1 we use
Gauss Elimination by segment as in [4], then $\mathcal{H}_{\Lambda}$ is an orthonormal symmetry adapted basis of $\Lambda_{\downarrow}$.

With this construction we reproved that $\Lambda_{\downarrow}$ is invariant. The above approach to computing a basis of $\Lambda_{\downarrow}$ is advantageous in two ways. First Gaussian elimination is performed only on smaller blocks. But also, when solving invariant and equivariant interpolation problems, the result will respect exactly the intended invariance or equivariance, despite possible numerical inaccuracy.

\subsection{Computing interpolants}

We consider an interpolation problem $(\Lambda, \phi)$ where $\Lambda$ is a $G$-invariant subspace of $\mathbb{K}[\mathrm{x}]^{*}$ and $\phi: \Lambda \rightarrow \mathbb{K}^{m}$. Take $\mathcal{P}$ to be a symmetry adapted basis of an invariant interpolation space $P$ for $\Lambda$ as obtained from Algorithm 1. The interpolant polynomial $p$ that solves $(\Lambda, \phi)$ in $P$ is given by

$$
p=\sum_{i=1}^{\mathbb{N}} \sum_{\alpha=1}^{n_{i}} \mathrm{~A}_{\mathrm{i}}^{-1} \phi\left(\mathcal{L}^{i, \alpha}\right)^{t}\left(\mathcal{P}^{i, \alpha}\right)^{t},
$$

where $\mathcal{P}^{i, \alpha}, \mathcal{L}^{i, \alpha}$ are as in (10) and $\mathrm{A}_{\mathrm{i}}=\mathrm{W}_{\mathcal{L}^{i, 1}}^{\mathcal{P}^{i, 1}}$. Note that we made no asumption on $\phi$. The invariance of $\Lambda$ allows to cut the problem into smaller blocks, independently of the structure of $\phi$. This illustrate how symmetry can be used to better organize computation : if we can choose the points of evaluation, the computational cost can be alleviated by choosing them with some symmetry.

When $\phi$ is invariant or equivariant, Equation (12) can be further reduced. If $(\Lambda, \phi)$ is an invariant interpolation problem, it follows from Remark 1 that $\phi\left(\mathcal{L}^{j}\right)=0$ for any $j>1$. Therefore for solving any invariant interpolation problem we only need to compute the first block of $\mathrm{W}_{\mathcal{L}}^{\mathcal{P}}$, i.e., the interpolant is given by $\mathrm{A}_{1}^{-1} \phi\left(\mathcal{L}^{1}\right)^{t}\left(\mathcal{P}^{1}\right)^{t}$.

More generally if $(\Lambda, \phi)$ is a $\vartheta-\theta$ equivariant problem, such that the irreducible representation $\rho_{i}$ does not occur in $\theta$, then $\phi\left(\mathcal{L}^{i}\right)=0$. The related block can thus be dismissed.

Example 5.3. Following on Example 3.2. Since we are interested in building an interpolation space for an invariant problem, we only need to compute bases of $\Lambda^{G}$ and $\mathbb{K}[\mathrm{x}]_{\leq 5}^{G}$. We have $\mathcal{L}^{G}=$ $\left\{\mathbb{e}_{\xi_{1}}, \sum_{i=2}^{6} \mathbb{e}_{\xi_{i}}, \sum_{i=7}^{11} \mathbb{e}_{\xi_{i}}, \sum_{i=12}^{16} \mathbb{e}_{\xi_{i}}\right\}$ and $\mathcal{P}^{G}=\left\{1, x_{1}^{2}+x_{2}^{2}, x_{1}^{4}+\right.$ $\left.2 x_{1}^{2} x_{2}^{2}+x_{2}^{4}, x_{1}^{5}-10 x_{1}^{3} x_{2}^{2}+5 x_{1} x_{2}^{4}\right\}$. Since $\mathrm{W}=\mathrm{W}_{\mathcal{L}^{G}}^{\mathcal{P}}$ is a square matrix with full rank, $\operatorname{span}_{\mathbb{K}}\left(\mathcal{P}^{G}\right)$ contains a unique invariant interpolant for any invariant interpolation problem. It has to be the least interpolant.

For $\phi$ given in Example 3.2, one finds the interpolant $p$ by solving the $5 \times 5$ linear system $\mathrm{W} a=\phi\left(\mathcal{L}^{G}\right)$. The solution $a=$ $(-0.3333333,3.295689,-36.59337,45.36692)^{t}$ provides the coefficients of $\mathcal{P}^{G}$ in $p$. The graph of $p$ is shown in Figure 1. If $p$ given above is only an approximation of the least interpolant, due to numerical inaccuracy, it is at least exactly invariant. Had we computed the least interpolant with the algorithm of [4], i.e., by elimination of the Vandermonde matrix based on the monomial basis, the least interpolant obtained would not be exactly invariant because of the propagation of numerical inacurracies.

We define the deviation from invariance (ISD) of $p=\sum_{\operatorname{deg} \alpha \leq 5} a_{\alpha} x^{\alpha}$ as $\sigma\left(a_{20}, a_{02}\right)+\sigma\left(a_{40}, \frac{a_{22}}{2}, a_{04}\right)+\sigma\left(a_{50},-\frac{a_{32}}{10}, \frac{a_{14}}{5}\right)+\sum_{\beta \in \mathcal{B}}\left|a_{\beta}\right|$ where $\sigma$ is the standard deviation, and $\mathcal{B}$ represents the exponents of the monomials that do not belongs to any of the elements in $\mathcal{P}^{G}$. In 
Table 1 we show the ISD for the interpolant $p$ computed with different precisions. The obtained polynomials are somehow far from being $G$-invariant.

\begin{tabular}{|c|c|c|c|c|}
\hline \# Digits & 10 & 15 & 20 & 30 \\
\hline ISD & 72.9614 & 40.0289 & 6.0967 & $<10^{-9}$ \\
\hline
\end{tabular}

Table 1: ISD values for different digits of precision

In the same spirit, let us mention that the condition number of $\mathrm{W}_{\Lambda}^{M}$, where $M$ is the monomial basis of $\mathbb{K}[\mathrm{x}]_{\leq 5}$, is more than $10^{2}$ times the condition number of $\mathrm{W}_{\mathcal{L}^{G}}^{\mathcal{P}^{G}}$. This is an indicator that two additional digits of precision are lost in the computation.

Example 5.4. Following up on Example 4.4. Let $\theta$ be the permutation representation of $D_{3}$ in $\mathbb{R}^{3} . \theta$ decomposes into two irreducible representations, the trivial representation and the irreducible representation $\vartheta$ of dimension 2. Let $\phi: \Lambda \rightarrow \mathbb{R}^{3}$ a $\vartheta-\theta$ equivariant map determined by $\phi\left(\varrho_{1}\right)=(1,-1,5)^{t}$. For solving $(\Lambda, \phi)$ we need only consider the first and third block of the Vandermonde matrix computed in Example 4.4. The $\rho^{*}-\theta$ equivariant map that solve $(\Lambda, \phi)$ is $P=\left(p_{1}, p_{2}, p_{3}\right)$ with:

$$
\begin{gathered}
p_{1}:=\frac{705}{4256} x_{1}^{2}+\frac{135}{4256} x_{2}^{2}+\frac{31}{56} \sqrt{3} x_{1}+\frac{93}{56} x_{2}-\frac{15}{112} \sqrt{3} x_{1} x_{2} \\
p_{2}:=\frac{705}{4256} x_{1}^{2}+\frac{135}{4256} x_{2}^{2}+\frac{31}{56} \sqrt{3} x_{1}-\frac{93}{56} x_{2}+\frac{15}{112} \sqrt{3} x_{1} x_{2} \\
p_{3}:=-\frac{75}{2128} x_{1}^{2}+\frac{495}{2128} x_{2}^{2}-\frac{31}{28} \sqrt{3} x_{1} .
\end{gathered}
$$

In Figure 4 we show the image of $\mathbb{R}^{2}$ by $P$ and the tangency conditions imposed by $\phi$.

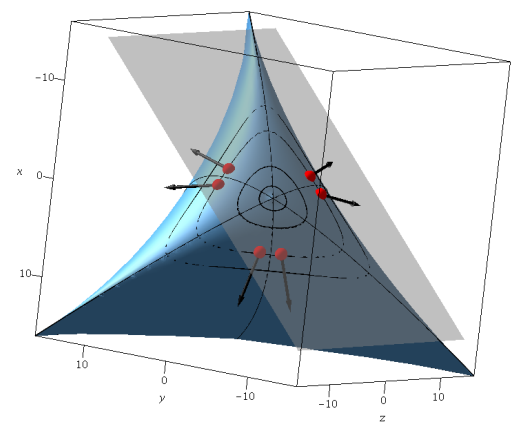

Figure 4: Parameterized surface with tangency constraints.

Example 5.5. Following up on Example 3.6. Since the representation $\vartheta$ of $D_{3}$ in $\mathbb{R}^{2}$ is irreducible, for computing any $\vartheta-\vartheta$ equivariant we only need to compute the third isotopic block in the Vandermonde matrix $\mathrm{W}_{\mathcal{L}^{3}}^{\mathcal{P}^{3}}$, where $\mathcal{P}$ is a basis for the interpolation space $P_{\Lambda}$ built by Algorithm 1. This block is $\mathrm{W}=\left(\begin{array}{cc}\mathrm{A}_{3} & \\ & \mathrm{~A}_{3}\end{array}\right)$. The rows correspond to

$\mathcal{L}^{3}:=\left[\mathcal{L}^{3,1}, \mathcal{L}^{3,2}\right], \mathcal{L}^{3,1}:=\left[\lambda_{1}, \lambda_{2}, \lambda_{3}, \lambda_{4}\right]$ and $\mathcal{L}^{3,2}:=\left[\lambda_{5}, \lambda_{6}, \lambda_{7}, \lambda_{8}\right]$ with $\lambda_{1}=\mathbb{e}_{\xi_{1}}+\mathbb{e}_{\xi_{2}}-\mathbb{e}_{\xi_{4}}-e_{\xi_{5}}$

$$
\lambda_{2}=\mathbb{e}_{\xi_{3}}-\mathbb{e}_{\xi_{4}}-\mathbb{e}_{\xi_{5}}+\mathbb{e}_{\xi_{6}},
$$$$
\lambda_{3}=\mathbb{e}_{\xi_{7}}+\mathbb{e}_{\xi_{8}}-\mathbb{e}_{\xi_{10}}-\mathfrak{e}_{\xi_{1} 1}
$$$$
\lambda_{4}=\mathbb{e}_{\xi_{9}}-\mathbb{e}_{\xi_{10}}-\mathbb{e}_{\xi_{1} 1}+\mathbb{e}_{\xi_{12}} \text {, }
$$

$$
\begin{aligned}
& \lambda_{5}=\frac{\sqrt{3}}{2}\left(-\mathbb{e}_{\xi_{1}}+\mathbb{e}_{\xi_{2}}+2 \mathbb{e}_{3}+\mathbb{e}_{\xi_{4}}-\mathbb{e}_{\xi_{5}}-2 \mathbb{e}_{\xi_{6}}\right), \\
& \lambda_{6}=\frac{\sqrt{3}}{2}\left(-2 \mathbb{e}_{\xi_{1}}+2 \mathbb{e}_{\xi_{2}}+\mathbb{e}_{\xi_{3}}-\mathbb{e}_{\xi_{4}}-\mathbb{e}_{\xi_{5}}-\mathbb{e}_{\xi_{6}}\right) . \\
& \lambda_{7}=\frac{\sqrt{3}}{2}\left(-\mathbb{e}_{\xi_{7}}+\mathbb{e}_{\xi_{8}}+2 \mathbb{e}_{\xi_{9}}+\mathbb{e}_{\xi_{10}}-\mathbb{e}_{\xi_{1} 1}-2 \mathbb{e}_{\xi_{12}}\right), \\
& \lambda_{8}=\frac{\sqrt{3}}{2}\left(-2 \mathbb{e}_{\xi_{7}}+2 \mathbb{e}_{\xi_{8}}+\mathbb{e}_{\xi_{9}}-\mathbb{e}_{\xi_{10}}-\mathbb{e}_{\xi_{1} 1}-\mathbb{e}_{\xi_{12}}\right) .
\end{aligned}
$$

The columns correspond to

$$
\begin{array}{r}
\mathcal{P}^{3}:=\left[\begin{array}{c}
\mathcal{P}^{3,1}:=\left[x, x^{2}-y^{2}, x^{3}+x y^{2}, x^{4}-y^{4}\right], \\
\mathcal{P}^{3,2}:=\left[y,-2 x y, y\left(x^{2}+y^{2}\right),-2 x y\left(x^{2}+y^{2}\right)\right]
\end{array}\right] . \\
A_{3}=-\frac{2}{27}\left(\begin{array}{cccc}
72 \sqrt{3} & -288 & 608 \sqrt{3} & -2432 \\
9 \sqrt{3} & 90 & 76 \sqrt{3} & 760 \\
45 \sqrt{3} & -90 & 140 \sqrt{3} & 280 \\
9 \sqrt{3} & 18 & 28 \sqrt{3} & 504
\end{array}\right)
\end{array}
$$

We thus determine that the equivariant interpolant for the interpolation problem described in Example 3.6 is given by :

$$
\begin{gathered}
p_{1}=\frac{\alpha}{320} x+\frac{3 \beta}{640}\left(x^{2}-y^{2}\right)+\frac{9 \gamma}{8960} x\left(x^{2}+y^{2}\right)+\frac{27 \delta}{17920}\left(x^{4}-y^{4}\right) \\
p_{2}=\frac{\alpha}{320} y-\frac{3 \beta}{320} x y+\frac{9 \gamma}{8960} y\left(x^{2}+y^{2}\right)-\frac{27 \delta}{8960} x y\left(x^{2}+y^{2}\right)
\end{gathered}
$$

where

$$
\alpha=\sqrt{3}(25 a-114 b)+494 d-185 c, \beta=\sqrt{3}(114 d-25 c)+38 b-5 a,
$$$$
\gamma=\sqrt{3}(42 b-25 a)+185 c-182 d, \delta=\sqrt{3}(25 c-42 d)+5 a-14 b .
$$

\section{REFERENCES}

[1] P. Chossat and R. Lauterbach. 2000. Methods in equivariant bifurcations and dynamical systems. World Scientific Publishing.

[2] M. Collowald and E. Hubert. 2015. A moment matrix approach to computing symmetric cubatures. (2015). https://hal.inria.fr/hal-01188290.

[3] C. De Boor and A. Ron. 1990. On multivariate polynomial interpolation. Constructive Approximation 6, 3 (1990)

[4] C. De Boor and A. Ron. 1992. Computational aspects of polynomial interpolation in several variables. Math. Comp. 58, 198 (1992).

[5] C. De Boor and A. Ron. 1992. The least solution for the polynomial interpolation problem. Mathematische Zeitschrift 210, 1 (1992).

[6] A. Fässler and E. Stiefel. 1992. Group theoretical methods and their applications. Springer.

[7] J.-C. Faugère and S. Rahmany. 2009. Solving systems of polynomial equations with symmetries using SAGBI-Gröbner bases. In Proc. ISSAC 2009. ACM, 151-158.

[8] J.-C. Faugere and J. Svartz. 2013. Grobner bases of ideals invariant under a commutative group: the non-modular case. In Proc. ISSAC 2013. ACM, 347-354.

[9] M. Gasca and T. Sauer. 2000. Polynomial interpolation in several variables. Advances in Computational Mathematics 12, 4 (2000), 377.

[10] K. Gatermann. 2000. Computer algebra methods for equivariant dynamical systems. Lecture Notes in Mathematics, Vol. 1728. Springer-Verlag, Berlin.

[11] K. Gatermann and P. A. Parrilo. 2004. Symmetry groups, semidefinite programs, and sums of squares. F. Pure Appl. Algebra 192, 1-3 (2004), 95-128.

[12] M. Golubitsky, I. Stewart, and D. G. Schaeffer. 1988. Singularities and groups in bifurcation theory. Vol. II. Applied Mathematical Sciences, Vol. 69. Springer.

[13] E. Hubert. 2019. Invariant Algebraic Sets and Symmetrization of Polynomial Systems. Fournal of Symbolic Computation (2019). DOI:10.1016/j.jsc.2018.09.002.

[14] E. Hubert and G. Labahn. 2012. Rational invariants of scalings from Hermite normal forms. In Proc. ISSAC 2012. ACM, 219-226.

[15] E. Hubert and G. Labahn. 2016. Computation of the Invariants of Finite Abelian Groups. Mathematics of Computations 85, 302 (2016), 3029-3050.

[16] T. Krick, A. Szanto, and M. Valdettaro. 2017. Symmetric interpolation, Exchange Lemma and Sylvester sums. Comm. Algebra 45, 8 (2017), 3231-3250.

[17] RA Lorentz. 2000. Multivariate Hermite interpolation by algebraic polynomials: A survey. Fournal of computational and applied mathematics 122, 1-2 (2000).

[18] C. Riener and M. Safey El Din. 2018. Real root finding for equivariant semialgebraic systems. In Proc. ISSAC 2018. ACM, 335-342.

[19] C. Riener, T. Theobald, L. J. Andrén, and J. B. Lasserre. 2013. Exploiting symmetries in SDP-relaxations for polynomial optimization. Math. Oper. Res. 38, 1 (2013).

[20] T. Sauer. 1998. Polynomial interpolation of minimal degree and Gröbner bases. London Mathematical Society Lecture Note Series (1998).

[21] J. P. Serre. 1977. Linear representations of finite groups. Springer.

[22] J. Verschelde and K. Gatermann. 1995. Symmetric Newton polytopes for solving sparse polynomial systems. Adv. in Appl. Math. 16, 1 (1995), 95-127. 